\title{
U.S. funding boosts accelerator project
}

Slated for completion in the year 2005 , the Large Hadron Collider will send pro tons into head-on collisions at higher energies than any previously achieved.

This week, U.S. officials agreed to help pay for the new particle accelerator, now under construction at the European Laboratory for Particle Physics (CERN) near Geneva. The agreement calls for a contribution of materials and services worth $\$ 531$ million over 8 years, which would cover about a tenth of the collider's estimated cost.

The decision to participate in the project marks the first time that the U.S. government has agreed to contribute significantly to the construction of an accelerator outside the United States. "Increasingly in fundamental research, no country can go it alone," says John H. Gibbons, the president's assistant for science and technology. "International collaborations have become an integral part of all our domestic science programs."

The U.S. contribution will speed completion of the collider by 3 years, says CERN's Christopher Llewellyn Smith.

Several hundred U.S. physicists have already formed collaborative groups to participate in designing and building the massive detectors, each about five stories tall, that will record particle paths and energies (SN: 4/6/96, p. 214). Eventually, a quarter of the U.S. experimental high-energy physics community may end up doing research at the facility, according to the Department of Energy.

Under the terms of the agreement, the Energy Department pledges to provide materials and services valued at $\$ 200$ million for the accelerator. Another $\$ 331$ million in components will be contributed to the detector effort.

The new collider is being built inside CERN's existing circular tunnel, about 27 kilometers in circumference, which originally housed an electron-positron collider. The design calls for radio-frequency energy to accelerate two beams of protons to nearly the speed of light. Powerful superconducting magnets would guide the beams around the ring.

When the collider's two adjacent proton beams, circulating in opposite directions, are brought together, protons will smash into each other at a combined energy of 14 teraelectronvolts. That's seven times the collision energy possible at the Fermi National Accelerator Laboratory's Tevatron collider, located near Batavia, Ill.

The CERN collider's energy should be high enough for researchers to investigate such questions as what physical mechanism or process determines the masses of the known fundamental particles. - - Peterson

PECEMBER 13, 1997 